\title{
Osteoarticular Cryptococcosis Successfully Treated with High-Dose Liposomal Amphotericin B Followed by Oral Fluconazole
}

This article was published in the following Dove Press journal: Infection and Drug Resistance

\author{
Guillem Deus' \\ Silvia Gómez-Zorrilla' \\ Daniel Echeverria-Esnal ${ }^{2}$ \\ Ana Siverio ${ }^{3}$ \\ Robert Güerri-Fernandez' \\ Jesús Ares ${ }^{4}$ \\ Nuria Campillo ${ }^{2}$ \\ Emili Letang ${ }^{1,5}$ \\ Hernando Knobel' \\ Santiago Grau ${ }^{2}$ \\ Juan Pablo Horcajada'
}

'Infectious Diseases Service, Hospital del Mar, Infectious Pathology and

Antimicrobials Research Group (IPAR), Institut Hospital del Mar d'Investigacions Mèdiques (IMIM), Universitat Autònoma de Barcelona (UAB), CEXS-Universitat Pompeu Fabra, Barcelona, Spain; ${ }^{2}$ Pharmacy Service, Hospital del Mar, Infectious Pathology and Antimicrobials Research Group (IPAR), Institut Hospital del Mar d'Investigacions Mèdiques (IMIM), Universitat Autònoma de Barcelona (UAB), Barcelona, Spain; ${ }^{3}$ Microbiology Service, Laboratori de Referència de Catalunya, Hospital del Mar, Barcelona, Spain; ${ }^{4}$ Department of Radiology, Hospital del Mar, Universitat Autònoma de Barcelona (UAB), Barcelona, Spain; ${ }^{5}$ ISGlobal, Barcelona Institute for Global, Universitat de BarcelonaHealth, Barcelona, Spain

Correspondence: Silvia Gómez-Zorrilla Hospital del Mar, Infectious Diseases Department, Passeig Maritim 25-29 I, Barcelona, 08003, Spain

Tel +34932483468

Fax +3493248325

Email sgomezzorrilla@psmar.cat
Background: Skeletal involvement of Cryptococcus neoformans is infrequent and usually associated with disseminated cryptococcosis or underlying predisposing conditions. We present an atypical case of osteoarticular cryptococcosis in an immunocompetent patient.

Case Presentation: We herein report a case of bone and soft tissue cryptococcal infection in a 42-year-old male from Pakistan with well-controlled diabetes without other associated immunodeficiencies treated with antifungal therapy without surgical debridement. Furthermore, the patient developed toxidermia due to fluconazole use, so a fluconazole desensitization was performed. Therapeutic management also included the performance of therapeutic drug monitoring of fluconazole plasma concentrations.

Conclusion: To our knowledge, this is the first case of osteoarticular cryptococcosis treated with this treatment regimen. This strategy may be of interest to try to reduce hospital stay and associated complications.

Keywords: Cryptococcus neoformans, osteoarticular cryptococcosis, immunocompetent, liposomal amphotericin B, fluconazole desensitization

\section{Case Report}

A 42-year-old male from Pakistan was admitted to hospital in July 2018 complaining of chronic lumbar pain. The patient, who had been living in Barcelona since 2003, was recently diagnosed with type 2 diabetes mellitus, established with two hemoglobin A1C tests of over 6.5\%, and was well controlled on diet. He had no other medical conditions. He worked in public works water and sewer maintenance and claimed to have had no prior contact with animals. The patient reported a 6-month history of mechanical lumbar pain, without any previous trauma. The only symptoms reported during this time were $5 \mathrm{~kg}$ weight loss and 24-hr fever. After five months of pain, nodular lesions in the right gluteal region were noticed and antibiotic treatment was initiated by his general practitioner (amoxicillinclavulanate $1 \mathrm{~g} / 200 \mathrm{mg}$ TID for 10 days and thereafter ciprofloxacin $500 \mathrm{mg}$ BID for 10 days). Despite antibiotic treatment, pain and inflammatory signs increased and spontaneous drainage of the gluteal abscess occurred. A sample was taken for microbiological cultures and Cryptococcus neoformans was isolated. The patient was hospitalized for further study. Physical examination at admission revealed two indurated areas without inflammatory signs in the right gluteus and right lumbar area. The rest of the exploration was unremarkable. Laboratory tests showed renal 
and liver function values within the normal range (total bilirubin $0.36 \mathrm{mg} / \mathrm{dl}$, GOT $31 \mathrm{UI} / \mathrm{L}$, GPT 29 UI/L, FA 67 UI/L), hemoglobin $15.3 \mathrm{~g} / \mathrm{dl}$, white cell count 6940 cells/ $\mu \mathrm{L}$ (44.2\% neutrophils, 44.5\% lymphocyte). The plasma cryptococcal antigen latex agglutination test (CLAT) was positive (1:100), suggesting a disseminated infection. A lumbar puncture was performed with normal results, including a negative CLAT. Human immunodeficiency virus (HIV), primary immunodeficiencies and a lymphoproliferative disorder were ruled out. CD4 and CD8 counts were in the normal range. Except for diabetes, no other predisposing factors were found. Thoracoabdominal computed tomography ruled out lung involvement and revealed a gluteal abscess and a $3 \mathrm{~cm}$ lytic lesion on the right iliac crest (Figure 1). An ultrasound-guided fine-needle aspiration biopsy of the iliac crest was performed. No further surgical debridement was performed. The sample was inoculated on Polyvitex and Sabouraud agar plates (Biomèrieux ${ }^{\circledR}$ ) and incubated at $37^{\circ} \mathrm{C}$. Growth was observed after $72 \mathrm{~h}$ incubation and the strain was identified by matrix-assisted laser desorption ionizationtime of flight mass spectrometry (MALDI-TOF MS, BrukerDaltonics ${ }^{\circledR}$ ) as C. neoformans. Antifungal susceptibility testing was performed using the Sensititre YeastOne $^{\circledR}$ method, showing susceptibility to fluconazole

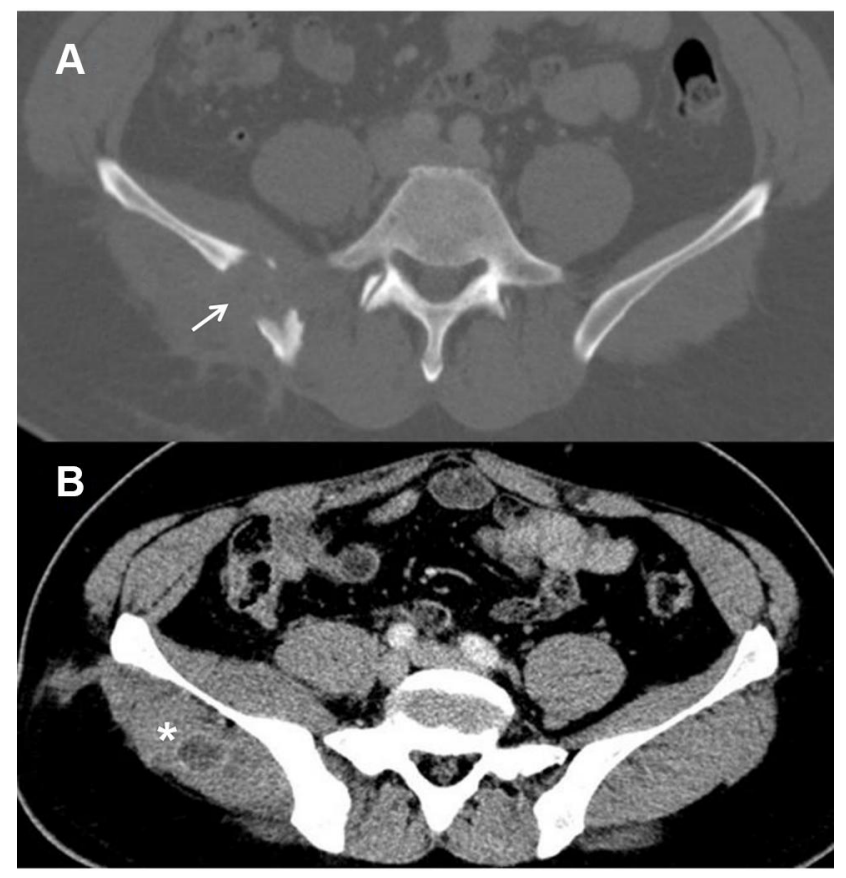

Figure I Transverse computed tomography image of the pelvis.

Notes: (A) Bone window: Osteolytic lesion with cortical bone destruction involving the right iliac bone (white arrow). (B) Soft tissue window: bulking of the right iliac and gluteus medius muscle with an abscess in the latter $\left(^{*}\right)$. [minimum inhibitory concentration (MIC) of $2 \mathrm{mg} / \mathrm{L}$ ] and amphotericin B.

Since the patient refused to remain in hospital due to family and work responsibilities, a treatment regimen was planned based on a single-dose of liposomal amphotericin B (L-AmB) $(10 \mathrm{mg} / \mathrm{kg}$, total dose $870 \mathrm{mg})$, infused over $3 \mathrm{~h}$, followed by outpatient therapy with oral fluconazole (800 mg/day). However, after the second dose of fluconazole, the patient developed a pruritic erythematous maculopapular rash. Given the lack of alternatives due to his personal circumstances and based on previous data in patients without HIV, ${ }^{1}$ a rapid oral desensitization protocol was performed (Table 1). Desensitization was conducted over 8 hours without complications, and the patient was discharged afterwards. He received $800 \mathrm{mg}$ fluconazole daily for 14 days, followed by $400 \mathrm{mg}$ daily for one year. The treatment was well tolerated with no adverse events. The pain resolved and plasma CLAT was undetectable at the end of treatment. During follow-up, on-site monitoring of fluconazole blood concentrations was conducted. While patient was on $800 \mathrm{mg}$, the area under the curve (AUC)/ MIC was 420 (objective >389). With the later $400 \mathrm{mg}$ dosage, a further 3 samples were obtained, yielding AUC/MICs of 144, 180 and 156 (goal >100). At the end of follow-up, a mild alteration in liver function was observed (total bilirubin $0.24 \mathrm{mg} / \mathrm{dl}$, AST $41 \mathrm{UI} / \mathrm{L}$, ALT $45 \mathrm{UI} / \mathrm{L}, \mathrm{GGT} 62 \mathrm{UI} / \mathrm{L}$ and FA $67 \mathrm{UI} / \mathrm{L})$, which was attributable to moderate liver steatosis confirmed by abdominal echography. After 20 months of follow-up, the patient continued asymptomatic without signs of recurrence.

\section{Discussion}

C. neoformans is a yeast normally found in soil. It causes opportunistic infection in immunocompromised patients, typically those with acquired immune deficiency syndrome. ${ }^{2}$ Although localized pulmonary disease is the main primary infection, extrapulmonary disease is common; especially central nervous system (CNS) infection. ${ }^{3}$ Osteoarticular cryptococcosis as the sole manifestation of extrapulmonary disease is rare, with an incidence of 5\% in patients with cryptococcal disease. ${ }^{3}$ Hematogenous dissemination after the primary pulmonary infection may explain bone affectation. ${ }^{3}$ All of these findings are even rarer in immunocompetent patients. ${ }^{4}$ In our case, diabetes mellitus was the only risk factor predisposing to cryptococcal infection. An exhaustive study ruled out other immunodeficiencies. Although diabetes mellitus is 
Table I Protocol of Fluconazole Desensitization

\begin{tabular}{|c|c|c|c|c|}
\hline Step & Fluconazole Dilution $\mathbf{( m g / m L})$ & Volume Administered $(\mathbf{m L})$ & Fluconazole Dose $(\mathbf{m g})$ & Cumulative Dose \\
\hline 1 & 0.002 & 10 & 0.02 & 0.02 \\
2 & 0.005 & 10 & 0.05 & 0.1 \\
3 & 0.01 & 10 & 0.2 & 0.17 \\
4 & 0.02 & 10 & 0.4 & 0.37 \\
5 & 0.04 & 10 & 0.8 & 0.77 \\
6 & 0.08 & 10 & 1.6 & 1.57 \\
7 & 0.16 & 10 & 4 & 3.17 \\
8 & 2.0 & 5 & 6.25 & 7.17 \\
9 & 2.0 & 3.13 & 12.5 & 13.42 \\
10 & 2.0 & 6.25 & 25 & 25.92 \\
11 & 2.0 & 12.5 & 50 & 50.92 \\
12 & 2.0 & 25 & 100 & 100.92 \\
13 & 2.0 & 50 & 200 & 200.92 \\
14 & - & - & 400 & 400.92 \\
15 & - & - & 800 & 800.92 \\
16 & - & - & 1600.92 \\
\hline
\end{tabular}

Note: A time interval of 30 minutes between doses was established.

a known risk factor for fungal infection, our patient had non-severe diabetes, with glucose levels well controlled by diet. We, therefore, considered low-grade immunosuppression due to diabetes. The patient also denied exposure to other risk factors, such as avian guano. While exceptional, other cases of cryptococcal infection with osteoarticular involvement in seemingly healthy patients have been reported. ${ }^{3-8}$

Little is known about the optimal treatment of osteoarticular cryptococcosis. Most cases reported in the literature propose a wide variety of treatments, based on antifungal drug therapy and surgical debridement. ${ }^{3,5}$ In contrast, more evidence is available for the management of CNS infection. Recently, a Phase 2 non-inferiority clinical trial in HIVassociated cryptococcal meningitis showed that a single high-dose of L-AmB plus high-dose oral fluconazole was non-inferior to 14 days of $3 \mathrm{mg} / \mathrm{kg} /$ day L-AmB in clearing cerebrospinal fluid infection. ${ }^{9}$ In our case, although CNS infection was ruled out, high levels of cryptococcal antigen in blood suggested disseminated cryptococcal infection. Moreover, bone involvement requires high doses of antifungal agents due to its poor vasculature. Consequently, based on the latest IDSA guidelines ${ }^{10}$ and the evidence in CNS infection, ${ }^{11}$ we treated this case with induction therapy consisting of a single high-dose of L-AmB followed by maintenance treatment with oral fluconazole for twelve months. Remarkably, we report a successful desensitization therapy that may be useful in other patients with fluconazole allergy. Throughout the entire course of fluconazole treatment, the plasma levels were considered therapeutic, since they achieved the recommended AUC/MIC of $\geq 389$ in induction therapy and $\mathrm{AUC} / \mathrm{MIC} \geq 100$ during consolidation therapy. ${ }^{12}$

In conclusion, we present an atypical case of bone and soft tissue cryptococcal infection in an immunocompetent host, which was managed with antifungal treatment without surgical debridement. Induction therapy with a single high-dose of L-AmB followed by maintenance treatment with oral fluconazole appears to be effective. Fluconazole desensitization and therapeutic drug monitoring ensured the proper application of treatment for twelve months and suggests that results may be similarly effective when applied to other patients.

\section{Abbreviations}

L-AmB, liposomal amphotericin B; CNS, central nervous system; CLAT, cryptococcal antigen latex agglutination test; HIV, human immunodeficiency virus; MIC, minimum inhibitory concentration; AUC, area under the curve.

\section{Data Sharing Statement}

All data (image and table) used in this study are included in this published article. We do not have metadata sharing, thus, it is not applicable to this article.

\section{Ethics Approval}

No institutional approval was required to publish the case details. 


\section{Consent for Publication}

The patient gave written informed consent to the publication of his history and photographs.

\section{Author Contributions}

All authors made a significant contribution to the work reported, whether that is in the conception, study design, execution, acquisition of data, analysis and interpretation, or in all these areas; took part in drafting, revising or critically reviewing the article; gave final approval of the version to be published; have agreed on the journal to which the article has been submitted; and agree to be accountable for all aspects of the work.

\section{Funding}

The authors thank the Spanish Society of Infectious Diseases and Clinical Microbiology (SEIMC) which supports the research of Dr. Silvia Gómez-Zorrilla.

\section{Disclosure}

Juan Pablo Horcajada reports being a speaker with honoraria advisory boards for MSD, Pfizer, and Menarini. The authors report no conflicts of interest in this work.

\section{References}

1. Randolph C, Kaplan C, Fraser B. Rapid desensitization to Fluconazole (Diflucan). Ann Allergy Asthma Immunol. 2008;100(6):616-617. doi:10.1016/S1081-1206(10)60063-4
2. Chayakulkeeree M, Perfect JR. Cryptococcosis. Infect Dis Clin North Am. 2006;20(3):507-544.

3. Medaris LA, Ponce B, Hyde Z, et al. Cryptococcal osteomyelitis: a report of 5 cases and a review of the recent literature. Mycoses. 2016;59(6):334-342.

4. Wood L, Miedzinski L. Skeletal cryptococcosis: case report and review of the literature. Can J Infect Dis. 1996;7:125-132.

5. Zhou HX, Lu L, Chu T, et al. Skeletal cryptococcosis from 1977 to 2013. Front Microbiol. 2015;5:740.

6. Ahn JH, Park C, Lee CW, Kim YC. Cryptococcal osteomyelitis of the first metatarsal head in an immunocompetent patient. $J$ Am Pediatr Med Assoc. 2017;107(3):248-252.

7. Sang J, Yang Y, Fan Y, et al. Isolated iliac cryptococcosis in an immunocompetent patient. PLoS Negl Trop Dis. 2018;12(3): e0006206.

8. Li Z, Liang J, Shen J, Qiu G, Weng X. Thoracolumbar scoliosis due to cryptococcal osteomyelitis: a case report and review of the literature. Medicine. 2016;95(5):e2613.

9. Jarvis JN, Leeme TB, Mooketsi M, et al. Short-course high-dose liposomal amphotericin $b$ for human immunodeficiency virus-associated cryptococcal meningitis: a phase 2 randomized controlled trial. Clin Infect Dis. 2019;68(3):393-401.

10. Perfect JR, Dismukes WE, Dromer F, et al. Clinical practice guidelines for the management of cryptococcal disease: 2010 update by the infectious diseases society of America. Clin Infect Dis. 2010;50 (3):291-322.

11. Molloy SF, Kanyama C, Heyderman RS, et al. Antifungal combinations for treatment of cryptococcal meningitis in Africa. $N$ Engl J Med. 2018;378(11):1004-1017.

12. Chesdachai S, Rajasingham R, Nicol MR, et al. Minimum inhibitory concentration distribution of fluconazole against cryptococcus species and the fluconazole exposure prediction model. Open Forum Infect Dis. 2019;6(10):ofz369.
Infection and Drug Resistance

\section{Publish your work in this journal}

Infection and Drug Resistance is an international, peer-reviewed openaccess journal that focuses on the optimal treatment of infection (bacterial, fungal and viral) and the development and institution of preventive strategies to minimize the development and spread of resistance. The journal is specifically concerned with the epidemiology of

\section{Dovepress}

antibiotic resistance and the mechanisms of resistance development and diffusion in both hospitals and the community. The manuscript management system is completely online and includes a very quick and fair peerreview system, which is all easy to use. Visit http://www.dovepress.com/ testimonials.php to read real quotes from published authors. 